\title{
Negombins A-I, New Chlorinated Polyfunctional Diterpenoids from the Marine Sponge Negombata sp.
}

\author{
Amira Rudi ${ }^{\dagger}$, Yehuda Benayahu ${ }^{\ddagger}$ and Yoel Kashman* \\ SUPPORTING INFORMATION
}

School of Chemistry, Raymond and Beverly Sackler Faculty of Exact Sciences and Department pf Zoology, Tel Aviv University, Ramat Aviv Tel Aviv 69978, ISRAEL

kashman@post.tau.ac.il

\footnotetext{
* To whom correspondence should be addressed. Phone: 972-3-6408419. Fax: 972-36409293

$\uparrow$ School of Chemistry

$\ddagger$ Department of Zoology
} 


\section{$\underline{\text { Table of Contents }}$}

Table 1. NMR Data of Negombins A - I (1-9) ..................................... S3

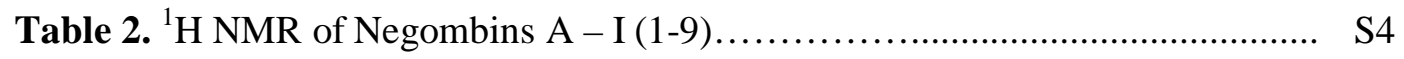

Figure S1. ${ }^{1} \mathrm{H}-\mathrm{NMR}$ spectrum of negombin $\mathrm{A}\left(\mathbf{1} ; 400 \mathrm{MHz}, \mathrm{CDCl}_{3}\right) \ldots \ldots \ldots \ldots \ldots \ldots . . . . . . . . . . .55$

Figure S2. ${ }^{13} \mathrm{C}-\mathrm{NMR}$ spectrum of negombin $\mathrm{A}\left(\mathbf{1} ; 100 \mathrm{MHz}, \mathrm{CDCl}_{3}\right) \ldots \ldots \ldots \ldots \ldots \ldots . . . . . . . . . .55$

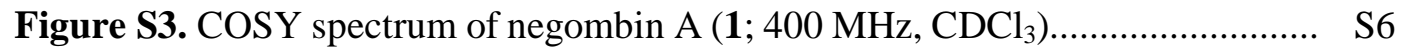

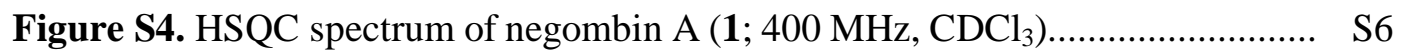

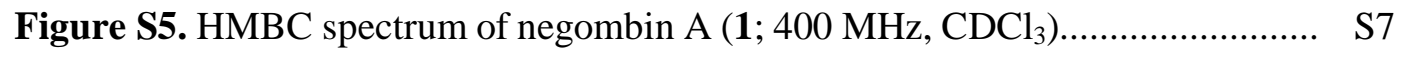

Figure S6. ${ }^{1} \mathrm{H}-\mathrm{NMR}$ spectrum of negombin $\mathrm{B}\left(2 ; 400 \mathrm{MHz}, \mathrm{CDCl}_{3}\right) \ldots \ldots \ldots \ldots \ldots \ldots . . . . . . . . . . .58$

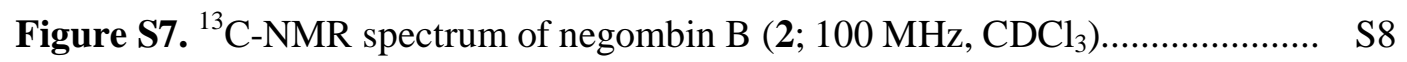

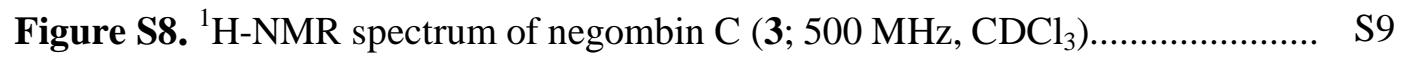

Figure S9. ${ }^{13} \mathrm{C}-\mathrm{NMR}$ spectrum of negombin $\mathrm{C}\left(\mathbf{3} ; 100 \mathrm{MHz}, \mathrm{CDCl}_{3}\right) \ldots \ldots \ldots \ldots \ldots \ldots . . . . . . . . .59$

Figure S10. ${ }^{1} \mathrm{H}-\mathrm{NMR}$ spectrum of negombin $\mathrm{D}\left(\mathbf{4} ; 400 \mathrm{MHz}, \mathrm{CDCl}_{3}\right) \ldots \ldots \ldots \ldots \ldots \ldots . \quad \mathrm{S} 10$

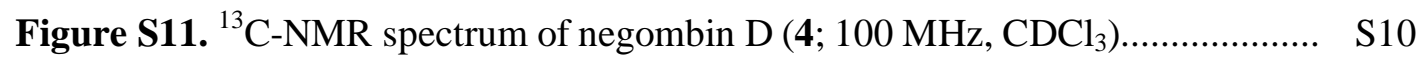

Figure S12. ${ }^{1} \mathrm{H}-\mathrm{NMR}$ spectrum of negombin $\mathrm{E}+\mathrm{F}\left(\mathbf{5}+\mathbf{6} ; 400 \mathrm{MHz}, \mathrm{CDCl}_{3}\right) \ldots \ldots \ldots \ldots . \quad \mathrm{S} 11$

Figure S13. ${ }^{13} \mathrm{C}-\mathrm{NMR}$ spectrum of negombin $\mathrm{E}+\mathrm{F}\left(\mathbf{5}+\mathbf{6} ; 100 \mathrm{MHz}, \mathrm{CDCl}_{3}\right) \ldots \ldots \ldots \ldots . \quad \mathrm{S} 11$

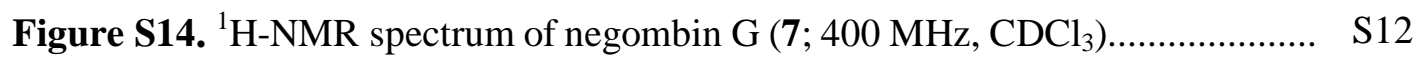

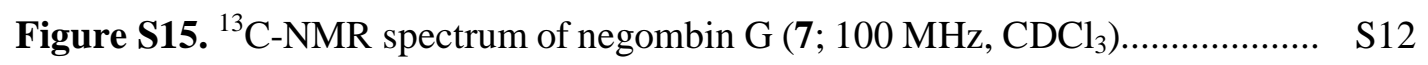

Figure S16. ${ }^{1} \mathrm{H}-\mathrm{NMR}$ spectrum of negombin $\mathrm{H}\left(\mathbf{8} ; 400 \mathrm{MHz}, \mathrm{CDCl}_{3}\right) \ldots \ldots \ldots \ldots \ldots \ldots . \quad \mathrm{S} 13$

Figure S17. ${ }^{13} \mathrm{C}-\mathrm{NMR}$ spectrum of negombin $\mathrm{H}\left(\mathbf{8} ; 100 \mathrm{MHz}, \mathrm{CDCl}_{3}\right) \ldots \ldots \ldots \ldots \ldots \ldots . . . . . . . . \quad \mathrm{S} 13$

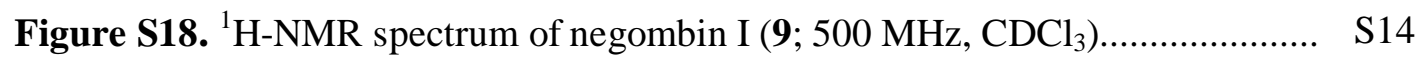

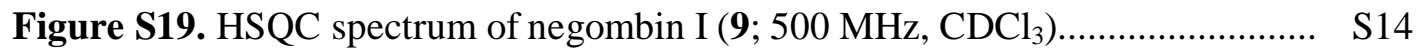


Table 1. ${ }^{13} \mathrm{C}$ NMR of Negombins A - I (1-9) $)^{a, b}$

\begin{tabular}{|c|c|c|c|c|c|c|c|c|}
\hline & 1 & 2 & 3 & 4 & $5(6)^{c}$ & 7 & 8 & 9 \\
\hline 1 & 72.0 & 72.1 & 40.4 & 73.2 & $82.9^{c}$ & 72.1 & 71.9 & 73.0 \\
\hline 2 & 35.3 & 35.4 & 28.9 & 35.7 & 32.4 & 35.2 & 35.2 & 35.2 \\
\hline 3 & 61.0 & 61.1 & 67.0 & 62.1 & 61.5 & 61.1 & 61.2 & 61.3 \\
\hline 4 & 41.6 & 41.5 & 41.9 & 41.7 & 42.8 & 41.4 & 41.6 & 42.1 \\
\hline 5 & 38.3 & 37.9 & 49.4 & 39.1 & 37.2 & 37.9 & 38.2 & 39.1 \\
\hline 6 & 25.8 & 25.0 & 18.7 & 24.4 & 23.7 & 24.9 & 24.8 & 24.7 \\
\hline 7 & 152.8 & 142.1 & 39.8 & 115.5 & 141.4 & 142.1 & 141.8 & 137.5 \\
\hline 8 & 138.2 & 139.9 & 70.2 & 136.2 & 140.0 & 142.0 & 142.0 & 143.0 \\
\hline 9 & 52.6 & 54.1 & 63.5 & 54.1 & 61.5 & 52.1 & 52.0 & 53.9 \\
\hline 10 & 41.7 & 41.6 & 37.8 & 37.6 & 40.6 & 41.7 & 42.4 & 37.6 \\
\hline 11 & 193.7 & 203.8 & 174.5 & 98.7 & $101.7^{c}$ & 203.8 & 203.6 & 98.5 \\
\hline 12 & 203.8 & 168.0 & 30.5 & 69.4 & 168.0 & 166.7 & 166.0 & 69.4 \\
\hline 13 & 15.5 & 15.1 & 17.5 & 14.8 & 17.6 & 15.1 & 15.0 & 14.7 \\
\hline 14 & 17.0 & 17.4 & 16.4 & 17.1 & 17.4 & 17.5 & 17.0 & 17.1 \\
\hline 15 & 38.2 & 37.8 & 38.2 & 38.0 & 37.4 & 34.5 & 39.8 & 34.6 \\
\hline 16 & 21.3 & 21.1 & 21.3 & 21.7 & 20.6 & 27.6 & 142.9 & 27.5 \\
\hline 17 & 123.7 & 123.6 & 123.7 & 124.0 & 125.5 & 76.3 & 120.3 & 79.0 \\
\hline 18 & 132.1 & 132.0 & 131.7 & 131.8 & 132.0 & 147.3 & 70.6 & 145.0 \\
\hline 19 & 25.9 & 25.0 & 25.7 & 25.7 & 25.6 & 111.3 & 29.9 & 115.0 \\
\hline 20 & 17.5 & 17.6 & 17.3 & 17.7 & 17.4 & 17.6 & 17.0 & 17.6 \\
\hline 21 & & 52.0 & 51.4 & & 52.1 & 54.1 & 53.9 & \\
\hline
\end{tabular}

\footnotetext{
${ }^{a}$ Data recorded in $\mathrm{CDCl}_{3}$ on Bruker Avance 400 instrument $(100 \mathrm{MHz})$.

${ }^{b}$ The $\mathrm{CH}$ correlations were assigned by an HSQC experiment.

${ }^{c}$ The only difference in the ${ }^{13} \mathrm{C}$ NMR of 6 are two peaks of C-9 and -11 at 83.4 and 97.0 , respectively.
} 
Table 2. ${ }^{1} \mathrm{H}$ NMR of Negombins A - I (1-9) $)^{a, b}$

\begin{tabular}{cccccccccc}
\hline & $\mathbf{1}$ & $\mathbf{2}$ & $\mathbf{3}$ & $\mathbf{4}$ & $\mathbf{5}$ & $\mathbf{6}^{\mathbf{c}}$ & $\mathbf{7}$ & $\mathbf{8}$ & $\mathbf{9}$ \\
\hline 1 & 3.68 & 3.68 & 1.401 .30 & 3.75 & 4.20 & 3.75 & 3.75 & 3.75 & 3.80 \\
2 & 2.252 .24 & 2.252 .25 & 2.081 .95 & 2.352 .20 & 2.402 .10 & & 2.252 .25 & 2.252 .25 & 2.302 .20 \\
3 & 4.55 & 4.40 & 4.02 & 4.52 & 4.40 & & 4.48 & 4.40 & 4.45 \\
5 & 1.95 & 1.90 & 1.12 & 1.90 & 1.95 & & 1.80 & 1.85 & 1.90 \\
6 & 2.522 .50 & 2.352 .35 & 1.801 .46 & 2.151 .98 & 2.122 .10 & & 2.202 .10 & 2.422 .40 & 2.202 .10 \\
7 & 7.05 & 7.22 & 1.901 .30 & 5.45 & 7.10 & 7.20 & 7.20 & 7.20 & 5.60 \\
9 & 3.50 & 3.45 & 2.10 & 2.72 & 2.60 & 2.75 & 3.50 & 3.50 & 2.80 \\
11 & 9.70 & 9.50 & & 5.30 & 5.22 & 5.61 & 9.50 & 9.55 & 5.30 \\
12 & 9.56 & & 1.12 & 4.504 .25 & - & & - & 9.50 & 4.604 .22 \\
13 & 1.00 & 1.00 & 1.20 & 0.80 & 0.90 & & 0.98 & 1.00 & 0.90 \\
14 & 1.05 & 0.95 & 0.90 & 1.00 & 1.05 & & 1.02 & 1.05 & 1.05 \\
15 & 1.621 .40 & 1.601 .30 & 1.651 .30 & 1.551 .30 & 1.651 .32 & & 1.301 .30 & 2.402 .05 & 1.401 .40 \\
16 & 2.021 .80 & 1.951 .80 & 1.901 .80 & 1.951 .80 & 1.981 .70 & & 1.601 .30 & $5.56 \mathrm{t}$ & 1.581 .30 \\
17 & 5.07 & 5.05 & 5.10 & 5.08 & 5.05 & & 4.00 & 5.75 & 3.86 \\
18 & - & - & - & - & & & - & - & - \\
19 & 1.69 & 1.70 & 1.70 & 1.68 & 1.70 & & 4.954 .88 & 1.31 & 5.105 .05 \\
20 & 1.61 & 1.50 & 1.62 & 1.65 & 1.60 & & 1.70 & 1.30 & 1.70 \\
21 & & 3.80 & 3.70 & & 3.72 & 3.70 & 3.75 & 3.80 &
\end{tabular}

${ }^{a}$ Data recorded in $\mathrm{CDCl}_{3}$ on Bruker Avance $500 \mathrm{MHz}$ instrument.

${ }^{b}$ The $\mathrm{CH}$ correlations were assigned by an HSQC experiment.

${ }^{c}$ All other resonance are the same as for $\mathbf{5}$. 
Figure S1. ${ }^{1} \mathrm{H}-\mathrm{NMR}$ spectrum of negombin A $\left(\mathbf{1} ; 400 \mathrm{MHz}, \mathrm{CDCl}_{3}\right)$

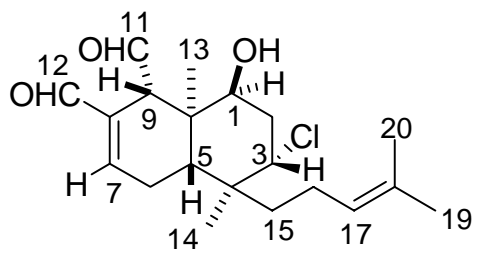
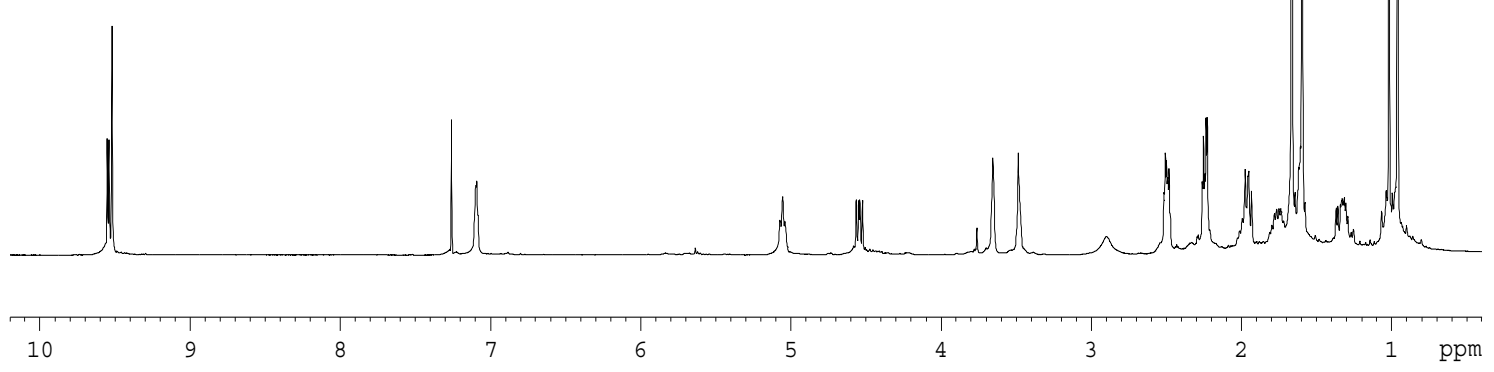

Figure S2. ${ }^{13} \mathrm{C}-\mathrm{NMR}$ spectrum of negombin A $\left(\mathbf{1} ; 100 \mathrm{MHz}, \mathrm{CDCl}_{3}\right)$
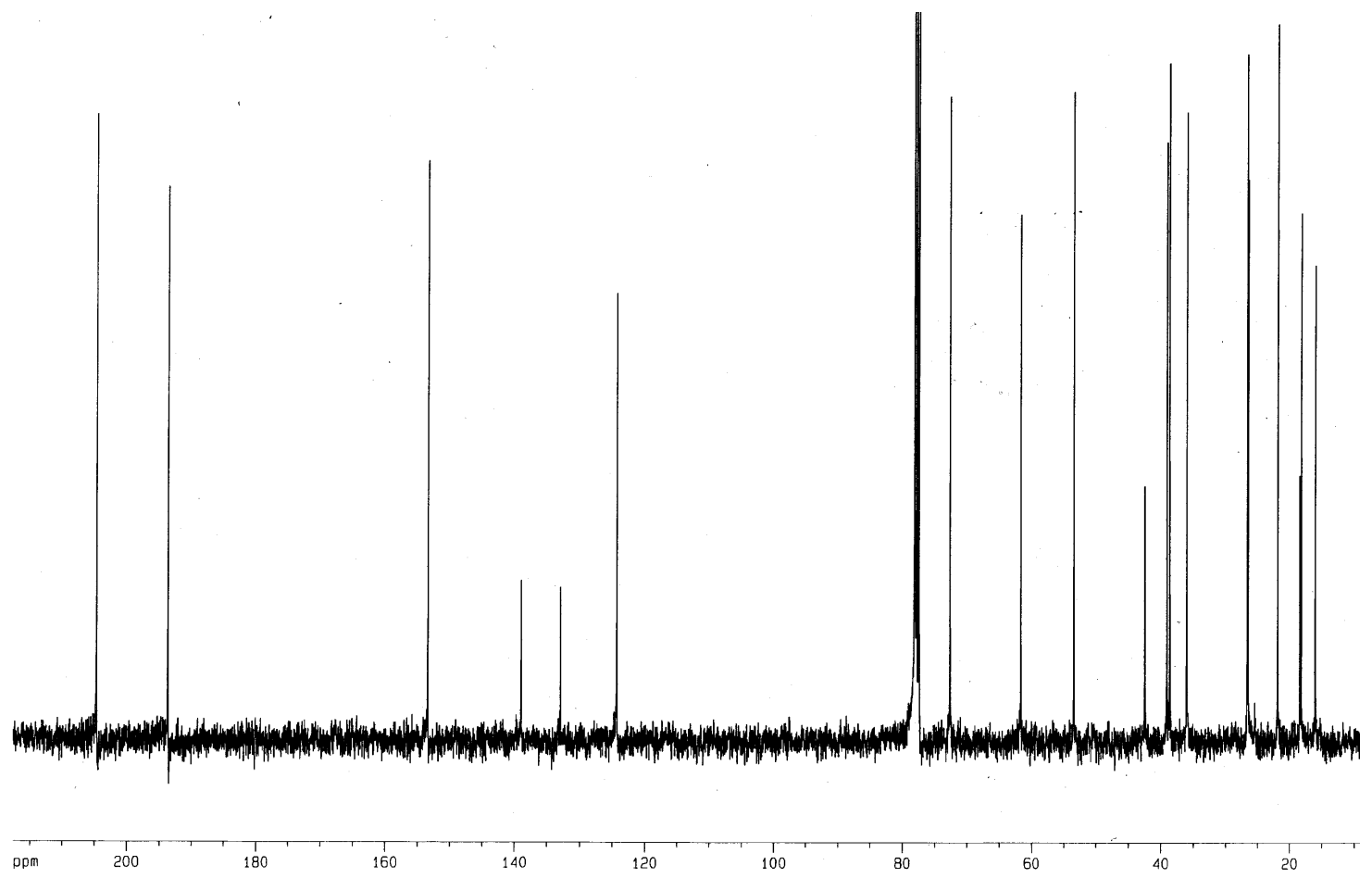

S5 
Figure S3. COSY spectrum of negombin A $\left(1 ; 400 \mathrm{MHz}, \mathrm{CDCl}_{3}\right)$

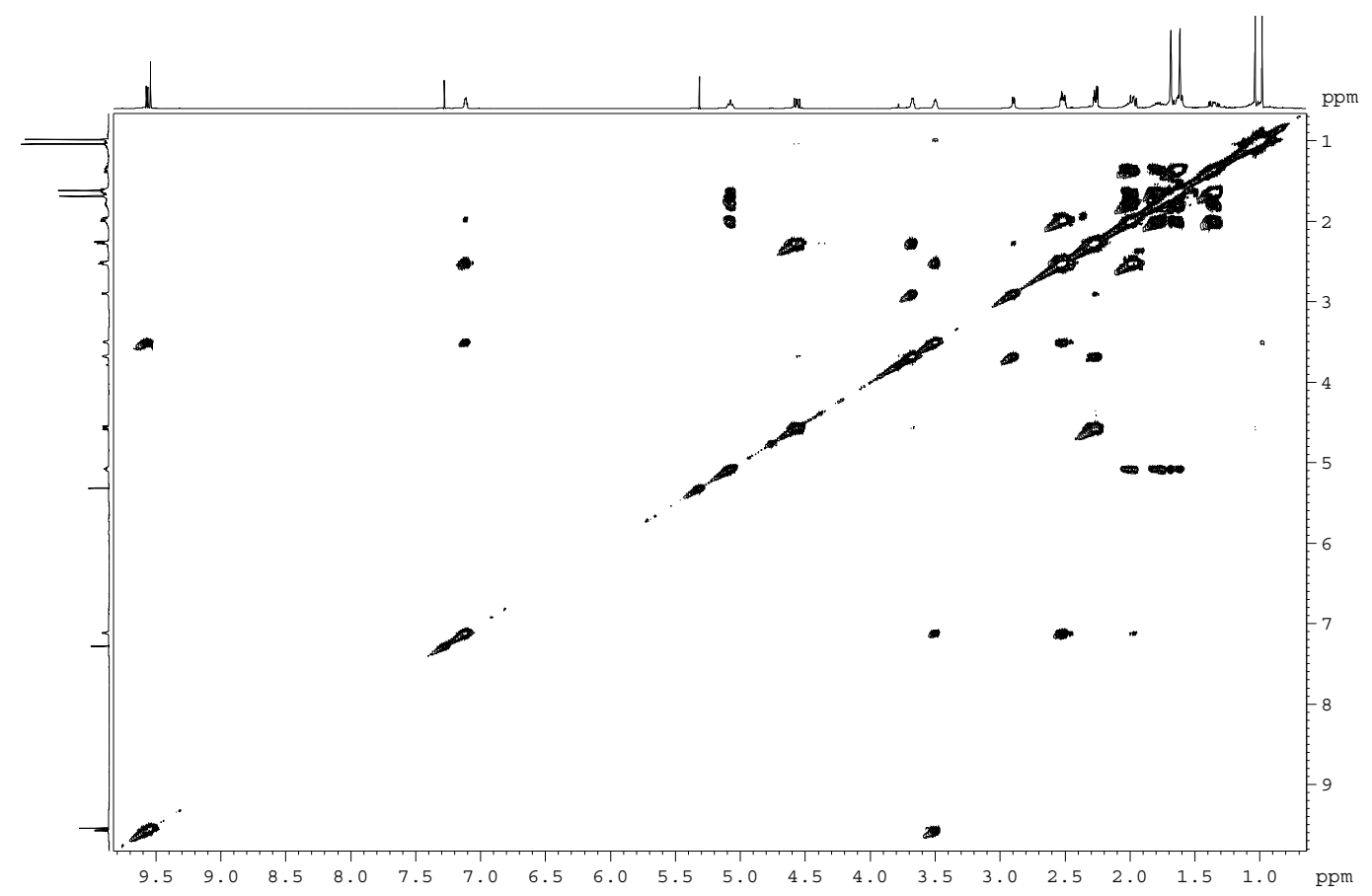

Figure S4. HSQC spectrum of negombin A $\left(\mathbf{1} ; 400 \mathrm{MHz}, \mathrm{CDCl}_{3}\right)$

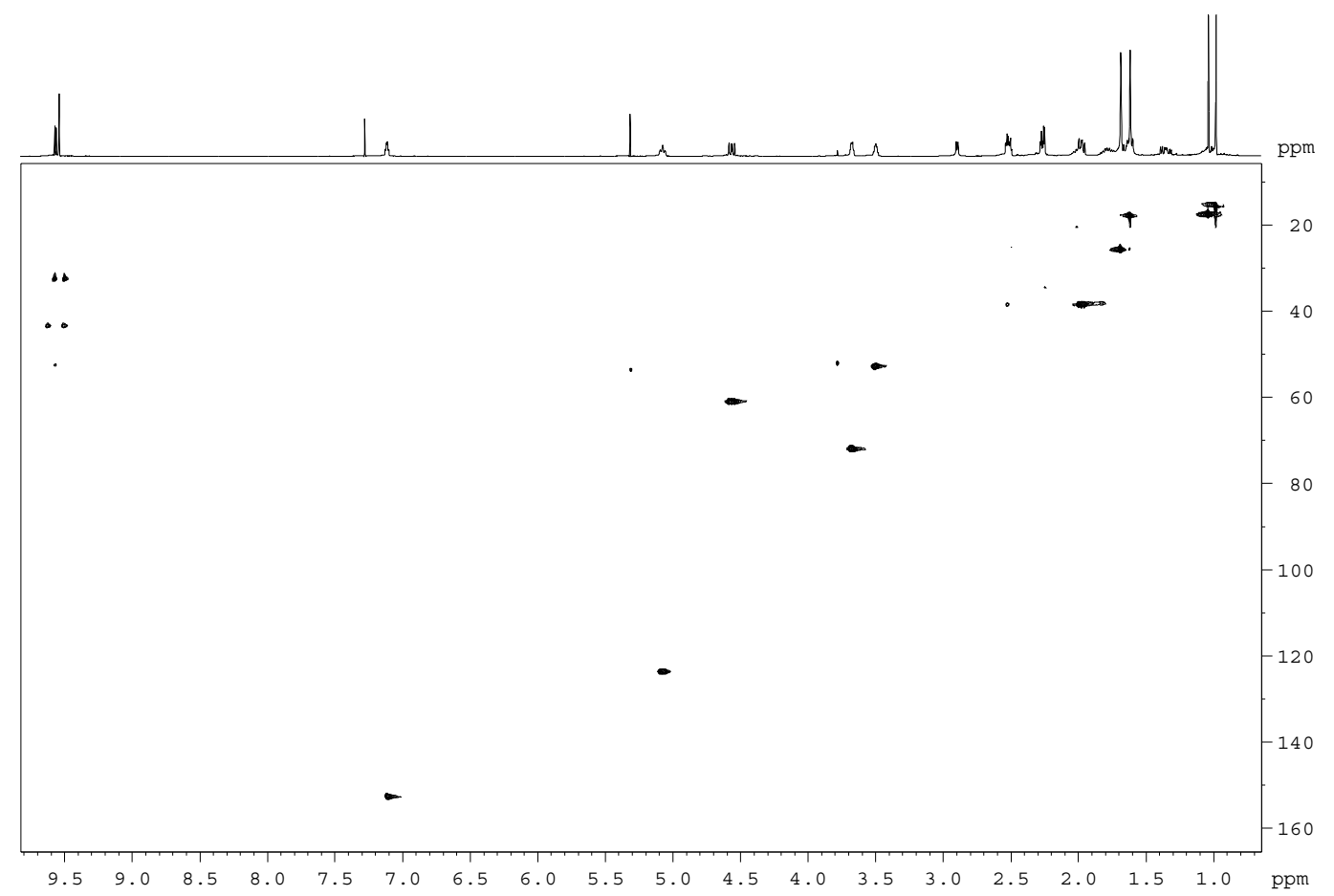

Figure S5. HMBC spectrum of negombin A (1; $\left.400 \mathrm{MHz}, \mathrm{CDCl}_{3}\right)$ 


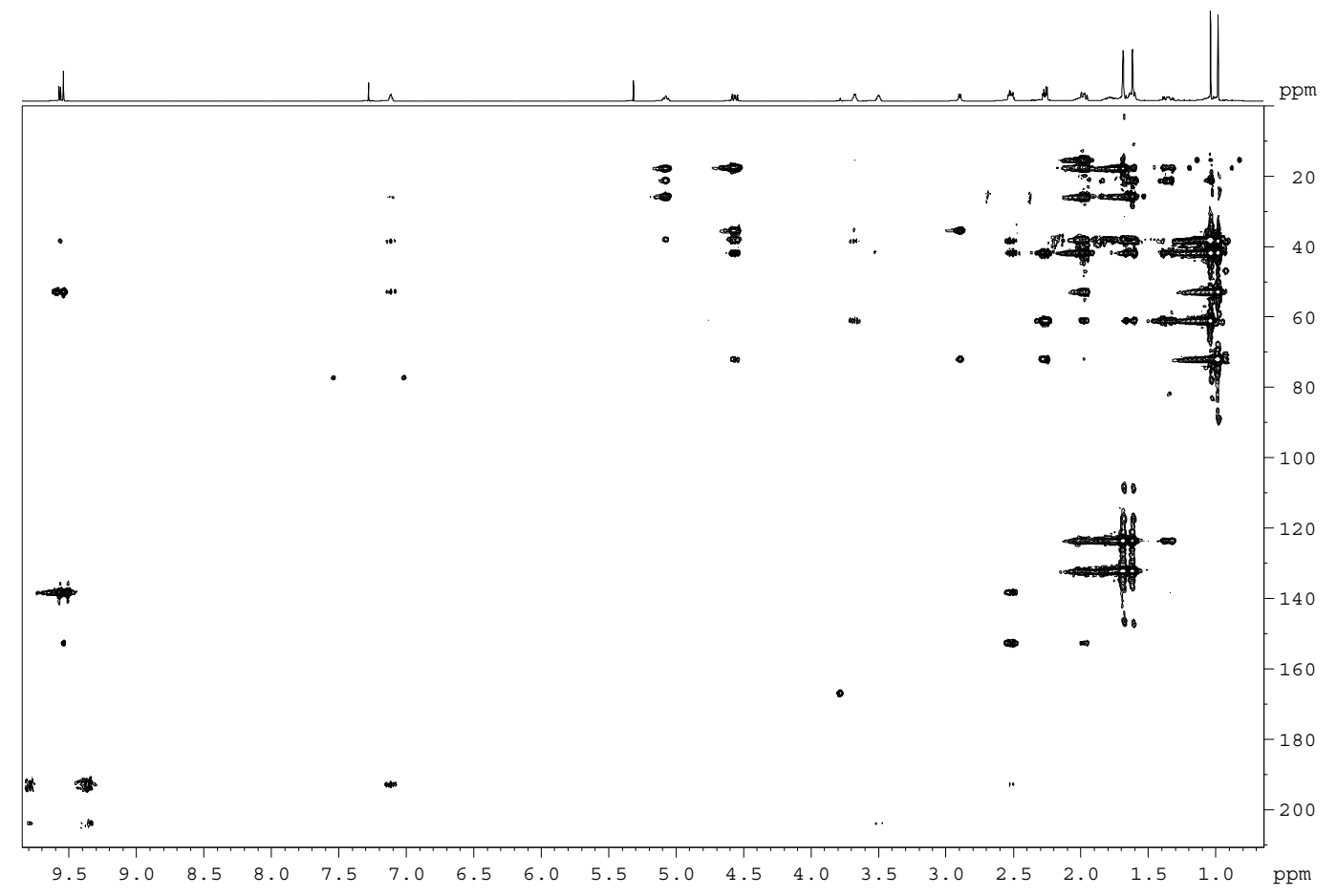


Figure S6. ${ }^{1} \mathrm{H}-\mathrm{NMR}$ spectrum of negombin $\mathrm{B}\left(\mathbf{2} ; 400 \mathrm{MHz}, \mathrm{CDCl}_{3}\right)$

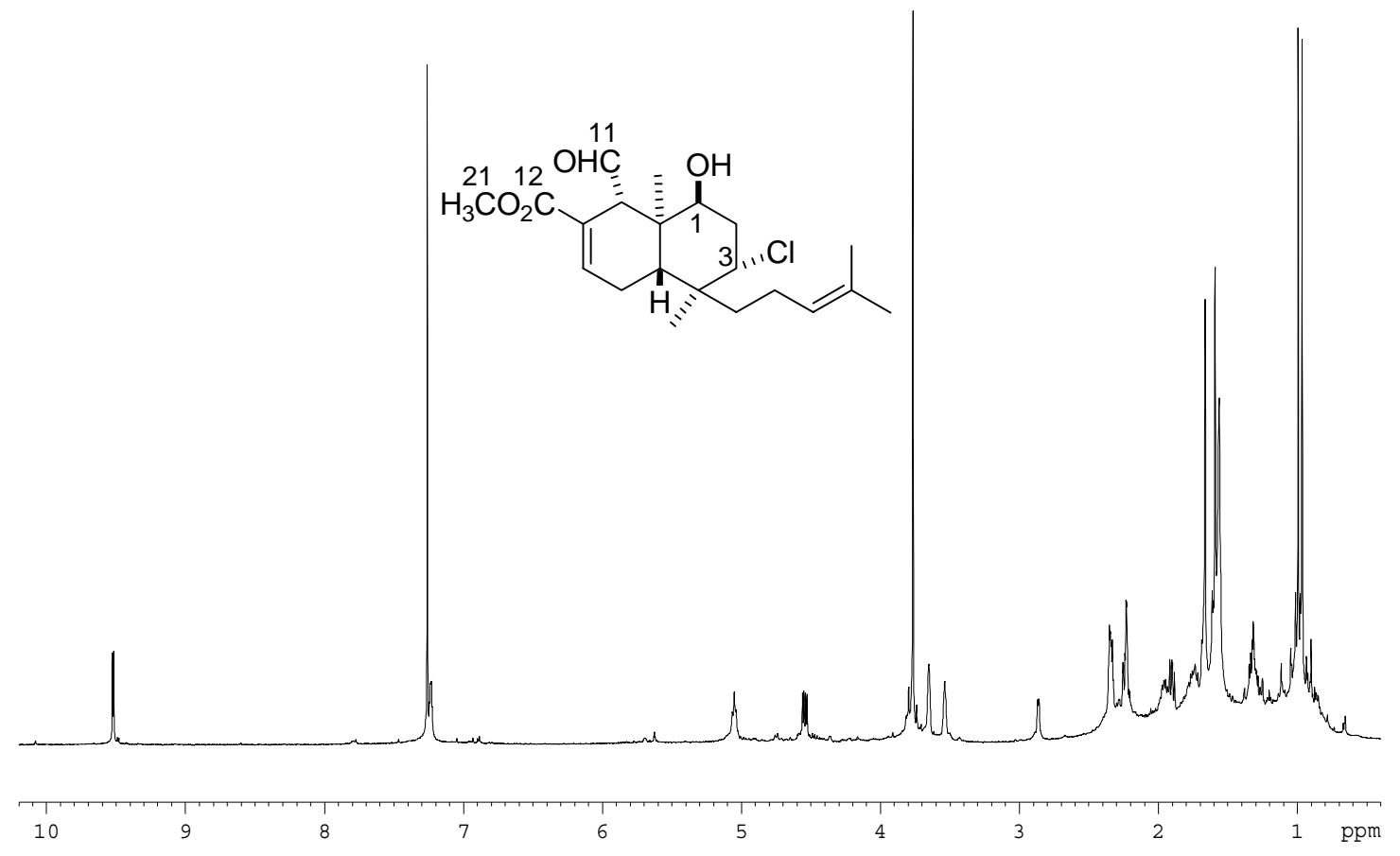

Figure S7. ${ }^{13} \mathrm{C}-\mathrm{NMR}$ spectrum of negombin $\mathrm{B}\left(\mathbf{2} ; 100 \mathrm{MHz}, \mathrm{CDCl}_{3}\right)$

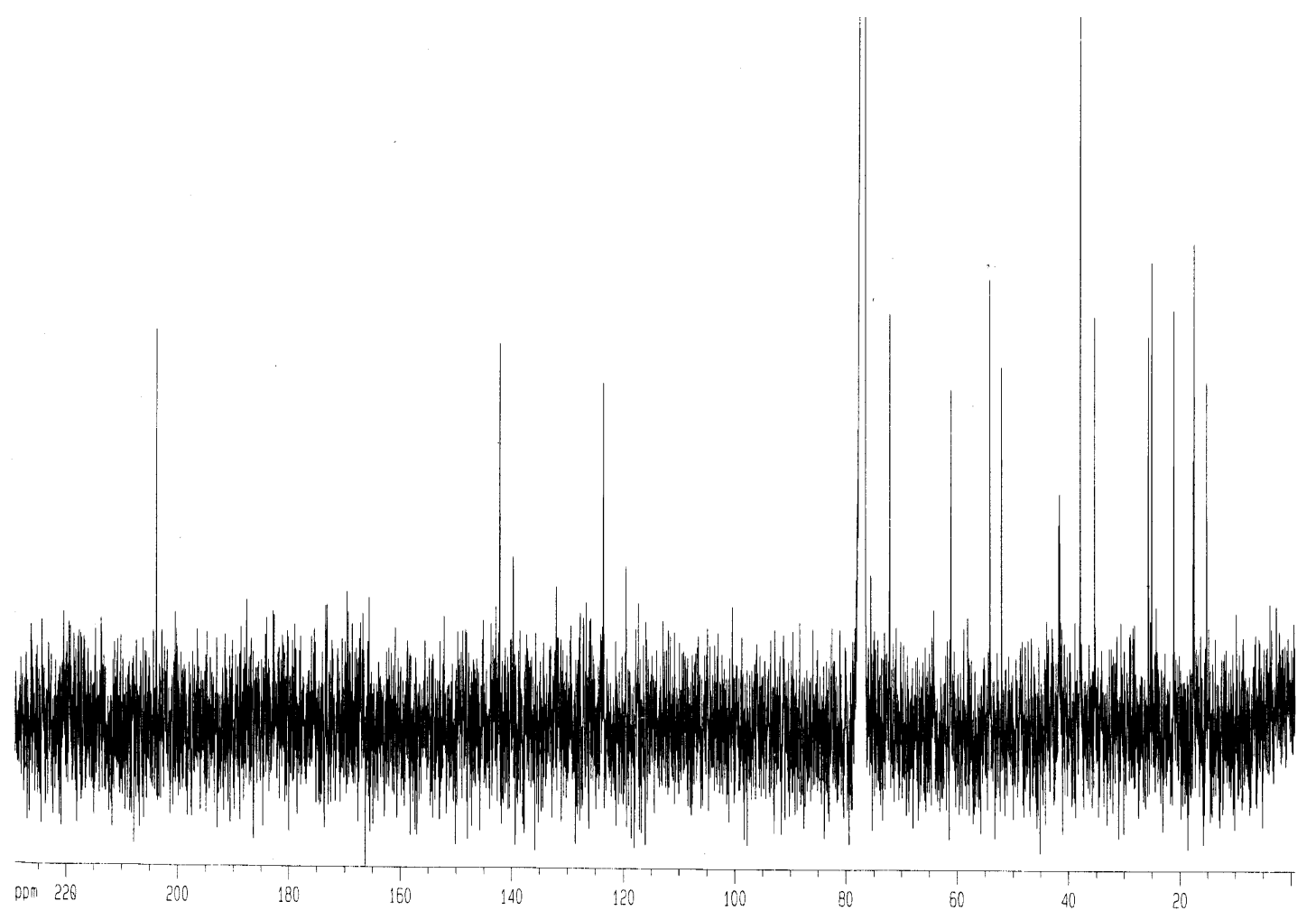


Figure S8. ${ }^{1} \mathrm{H}-\mathrm{NMR}$ spectrum of negombin $\mathrm{C}\left(\mathbf{3} ; 500 \mathrm{MHz}, \mathrm{CDCl}_{3}\right)$

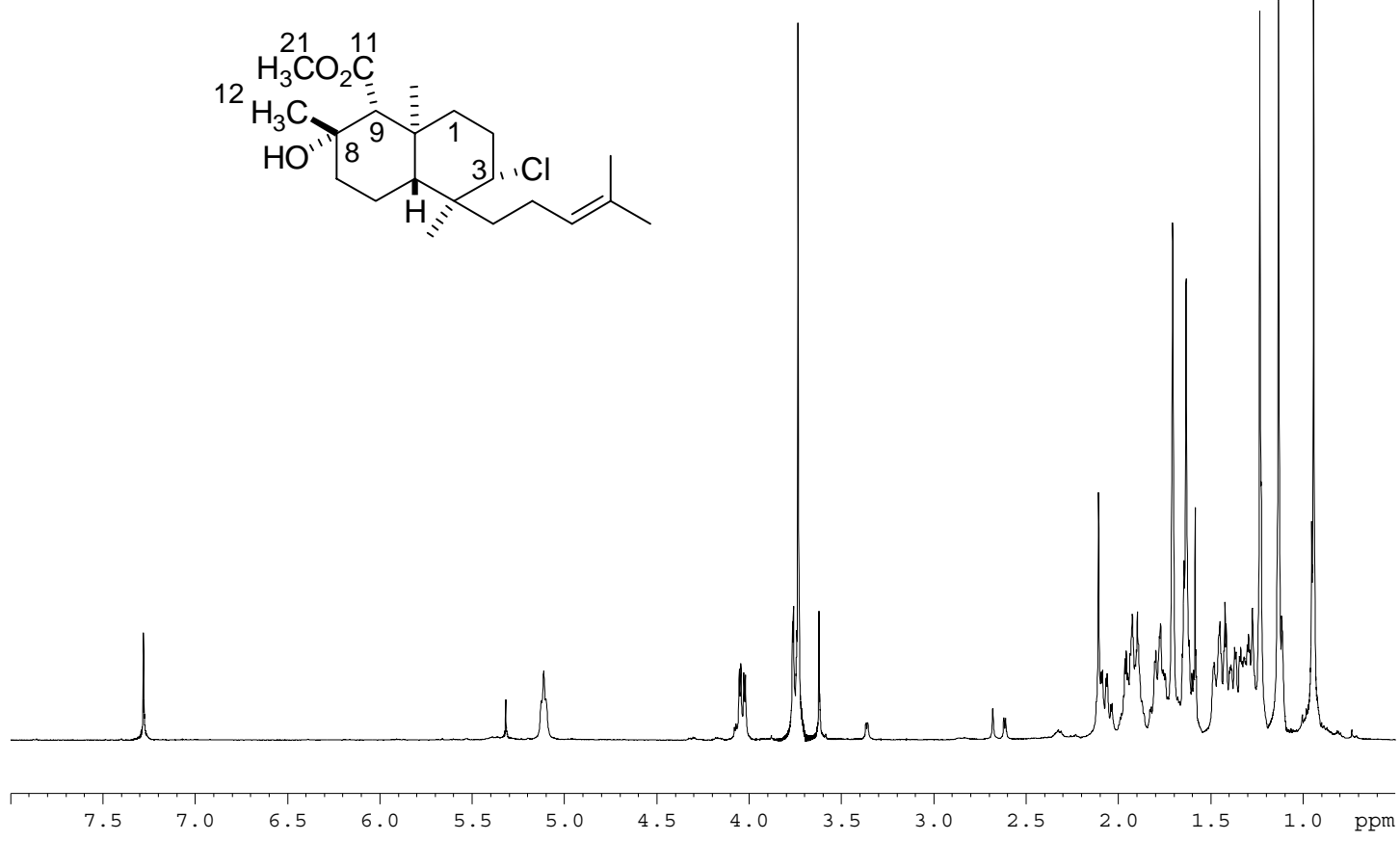

Figure S9. ${ }^{13} \mathrm{C}-\mathrm{NMR}$ spectrum of negombin $\mathrm{C}\left(\mathbf{3} ; 100 \mathrm{MHz}, \mathrm{CDCl}_{3}\right)$

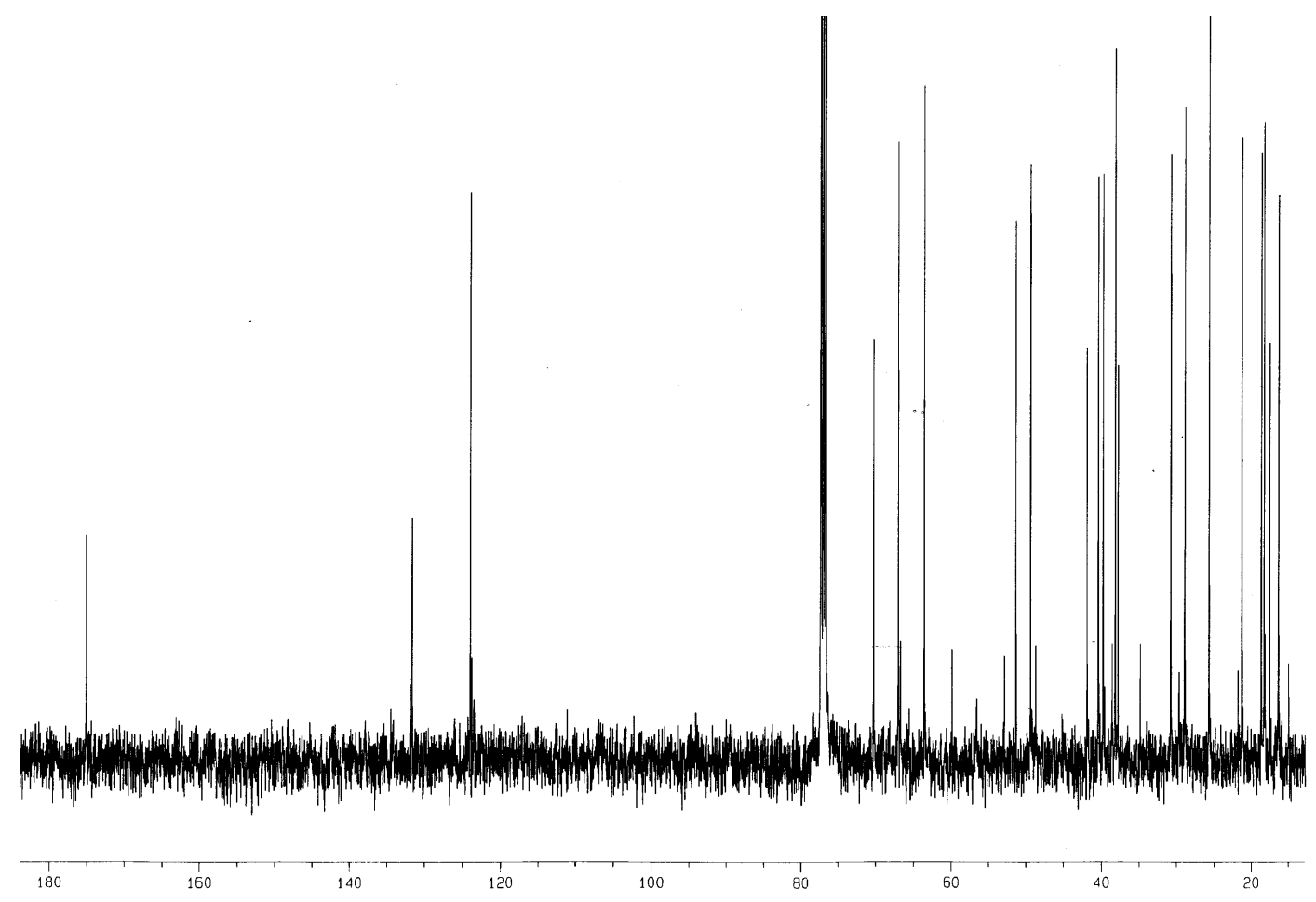

S9 
Figure S10. ${ }^{1} \mathrm{H}-\mathrm{NMR}$ spectrum of negombin $\mathrm{D}\left(\mathbf{4} ; 400 \mathrm{MHz}, \mathrm{CDCl}_{3}\right)$

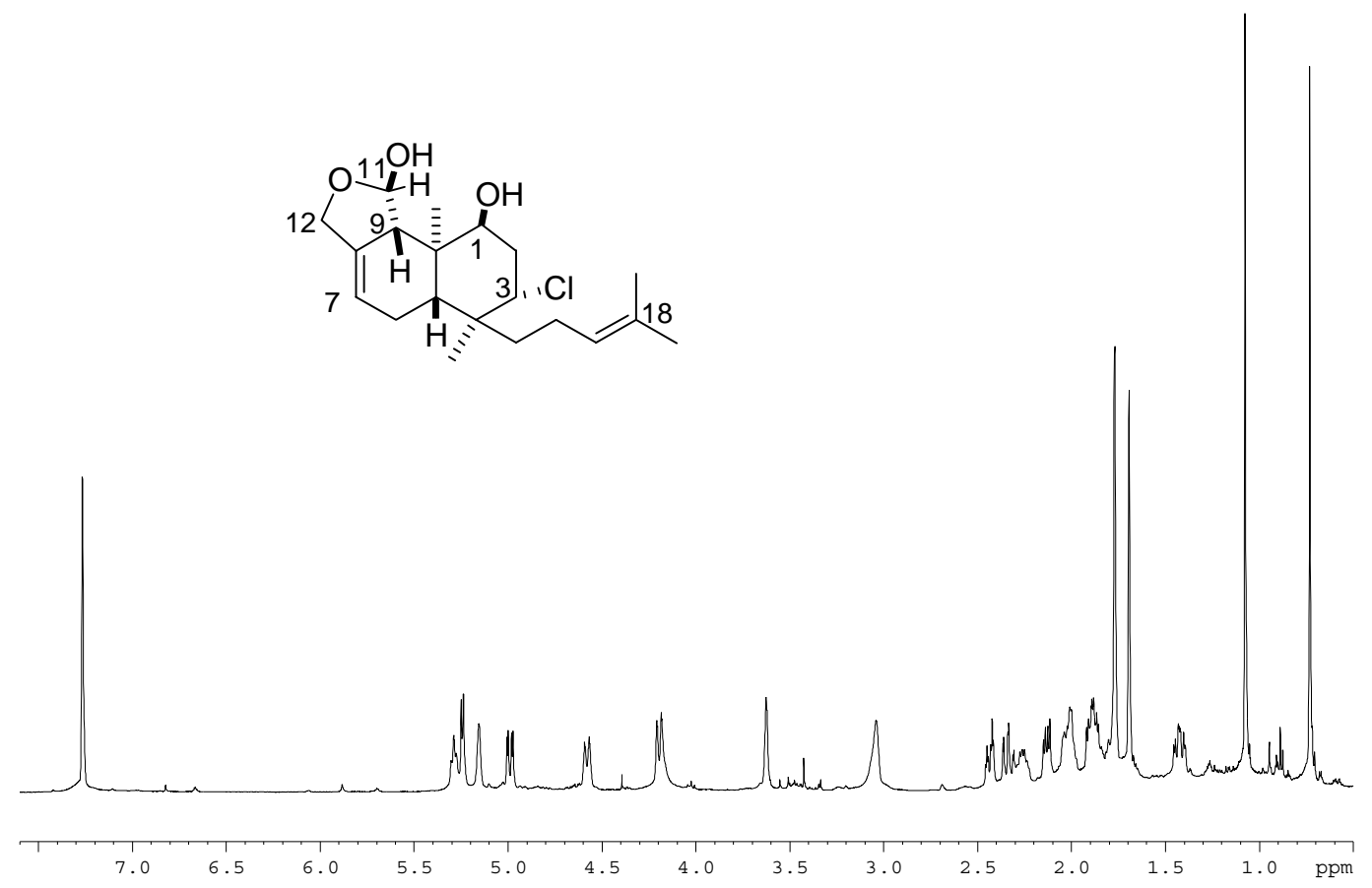

Figure S11. ${ }^{13} \mathrm{C}-\mathrm{NMR}$ spectrum of negombin $\mathrm{D}\left(\mathbf{4} ; 100 \mathrm{MHz}, \mathrm{CDCl}_{3}\right)$

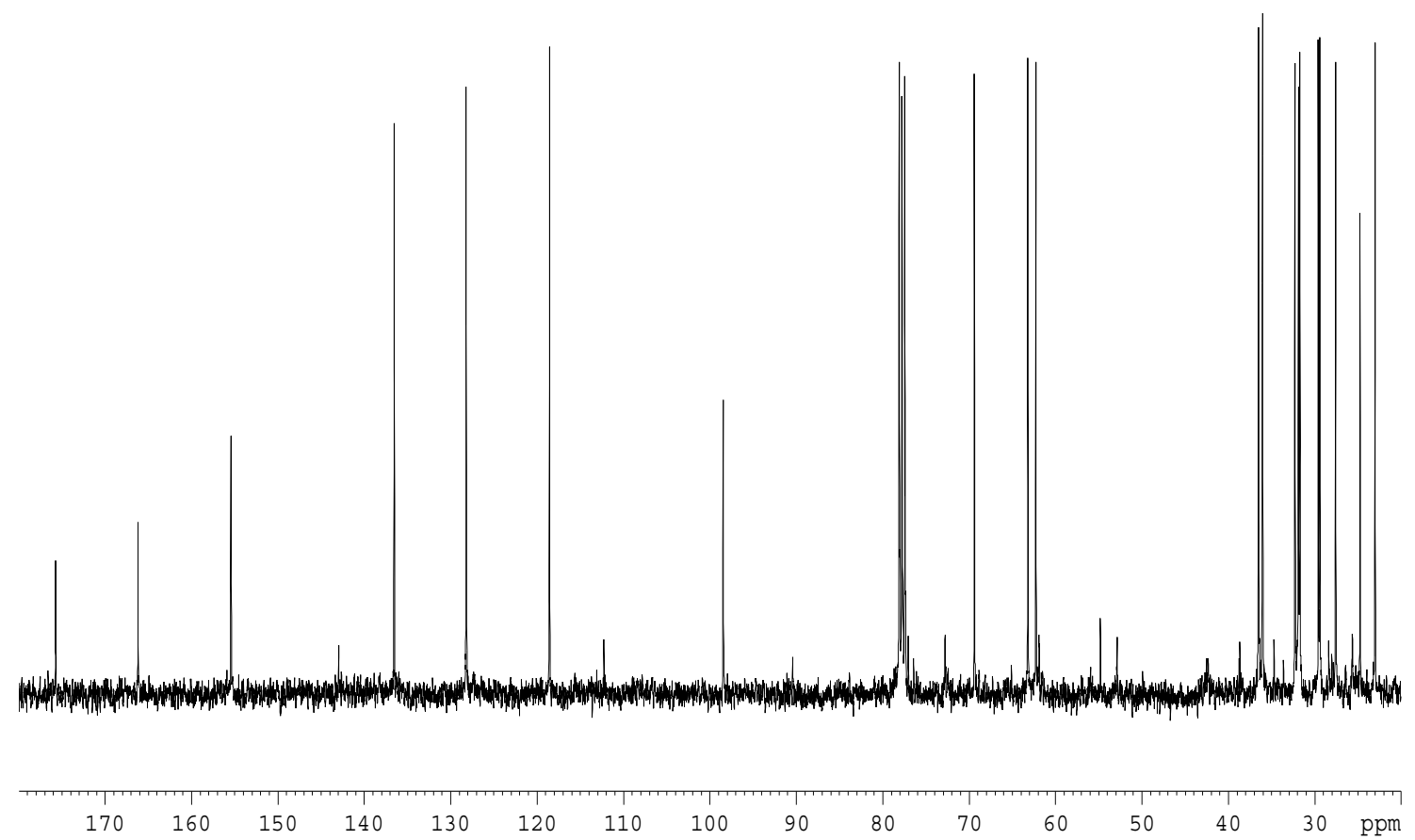


Figure S12. ${ }^{1} \mathrm{H}-\mathrm{NMR}$ spectrum of negombin $\mathrm{E}+\mathrm{F}\left(\mathbf{5}+\mathbf{6} ; 400 \mathrm{MHz}, \mathrm{CDCl}_{3}\right)$

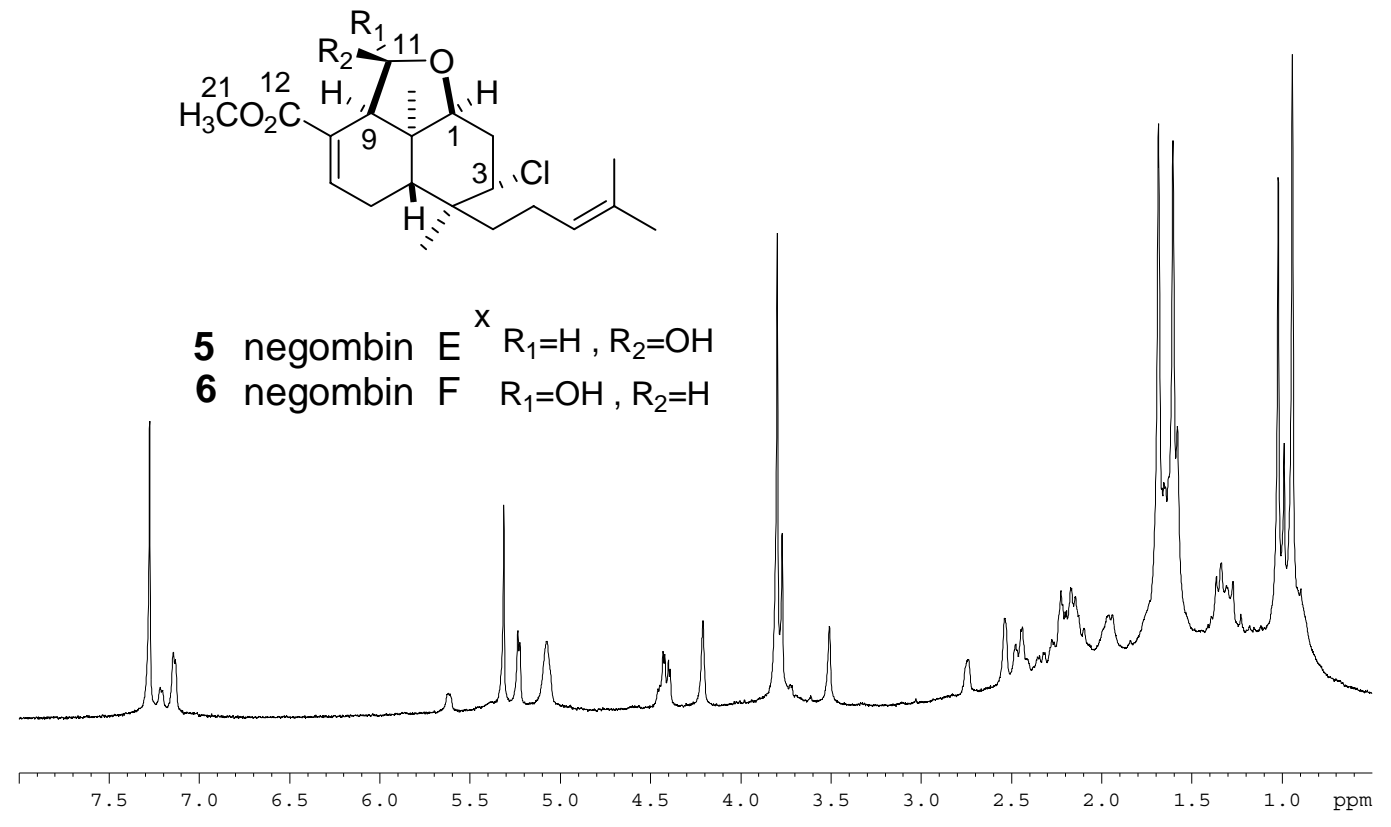

Figure S13. ${ }^{13} \mathrm{C}-\mathrm{NMR}$ spectrum of negombin $\mathrm{E}+\mathrm{F}\left(\mathbf{5}+\mathbf{6} ; 100 \mathrm{MHz}, \mathrm{CDCl}_{3}\right)$

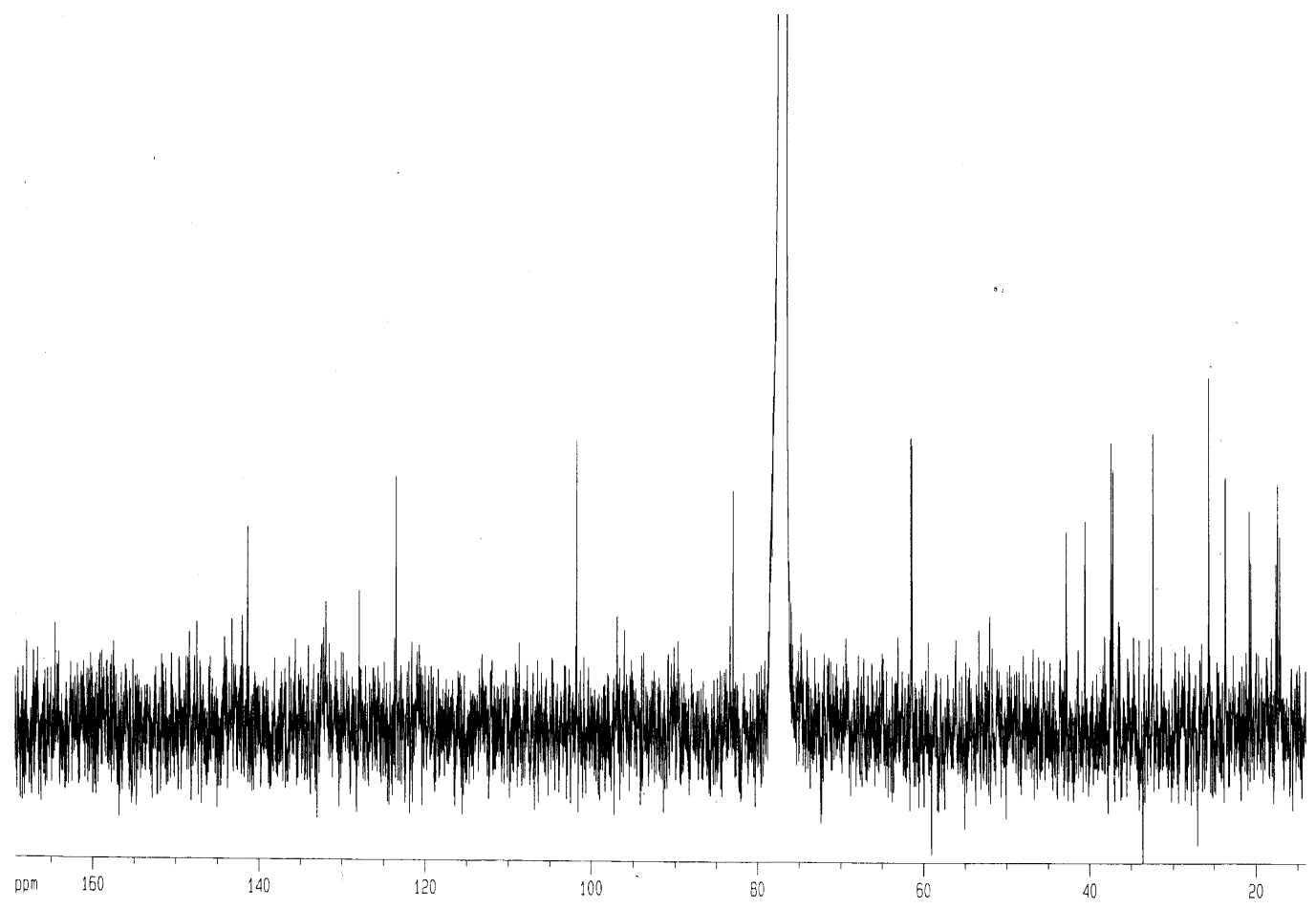


Figure S14. ${ }^{1} \mathrm{H}-\mathrm{NMR}$ spectrum of negombin $\mathrm{G}\left(\mathbf{7} ; 400 \mathrm{MHz}, \mathrm{CDCl}_{3}\right)$

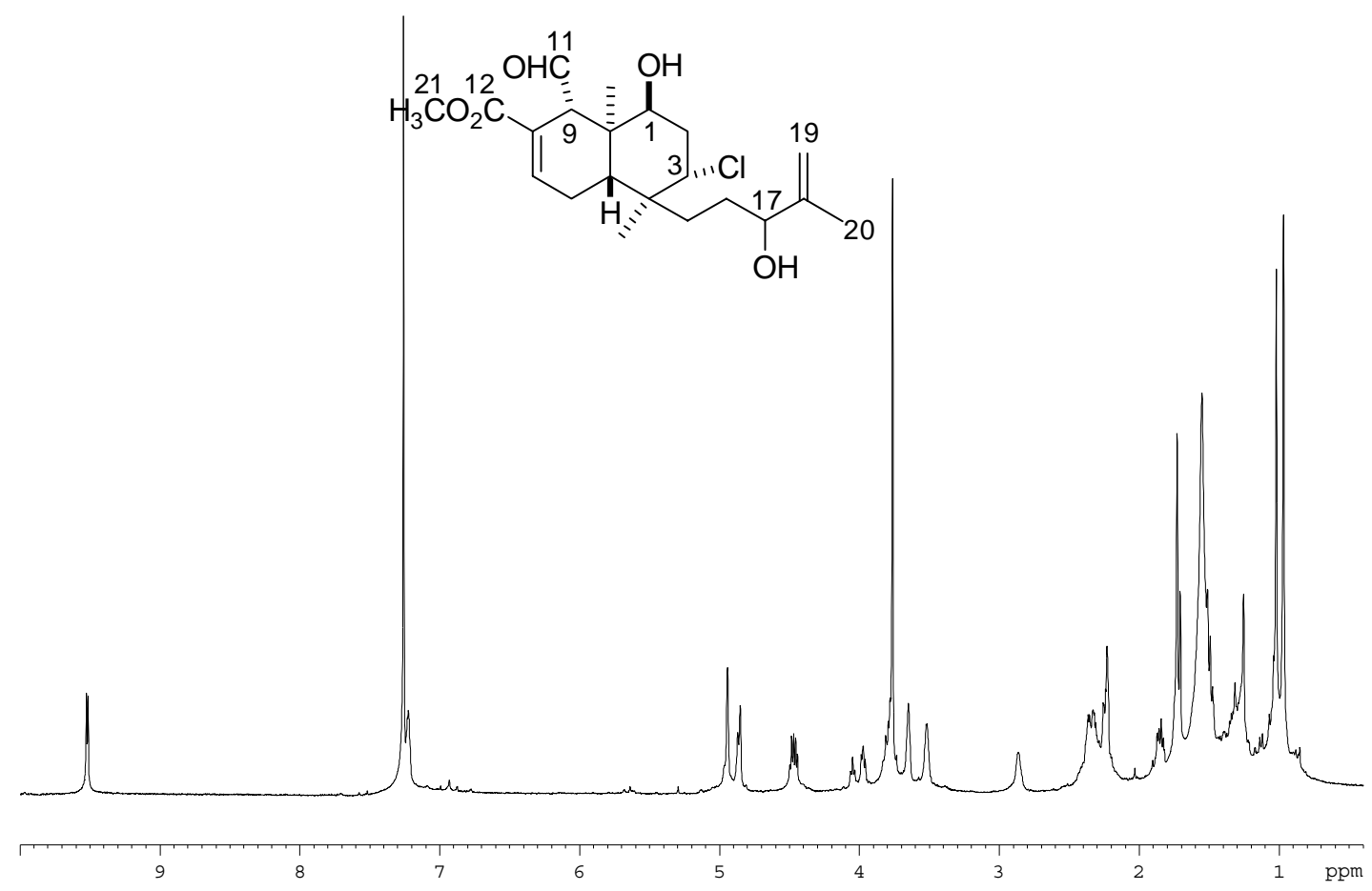

Figure S15. ${ }^{13} \mathrm{C}-\mathrm{NMR}$ spectrum of negombin $\mathrm{G}\left(\mathbf{7} ; 100 \mathrm{MHz}, \mathrm{CDCl}_{3}\right)$

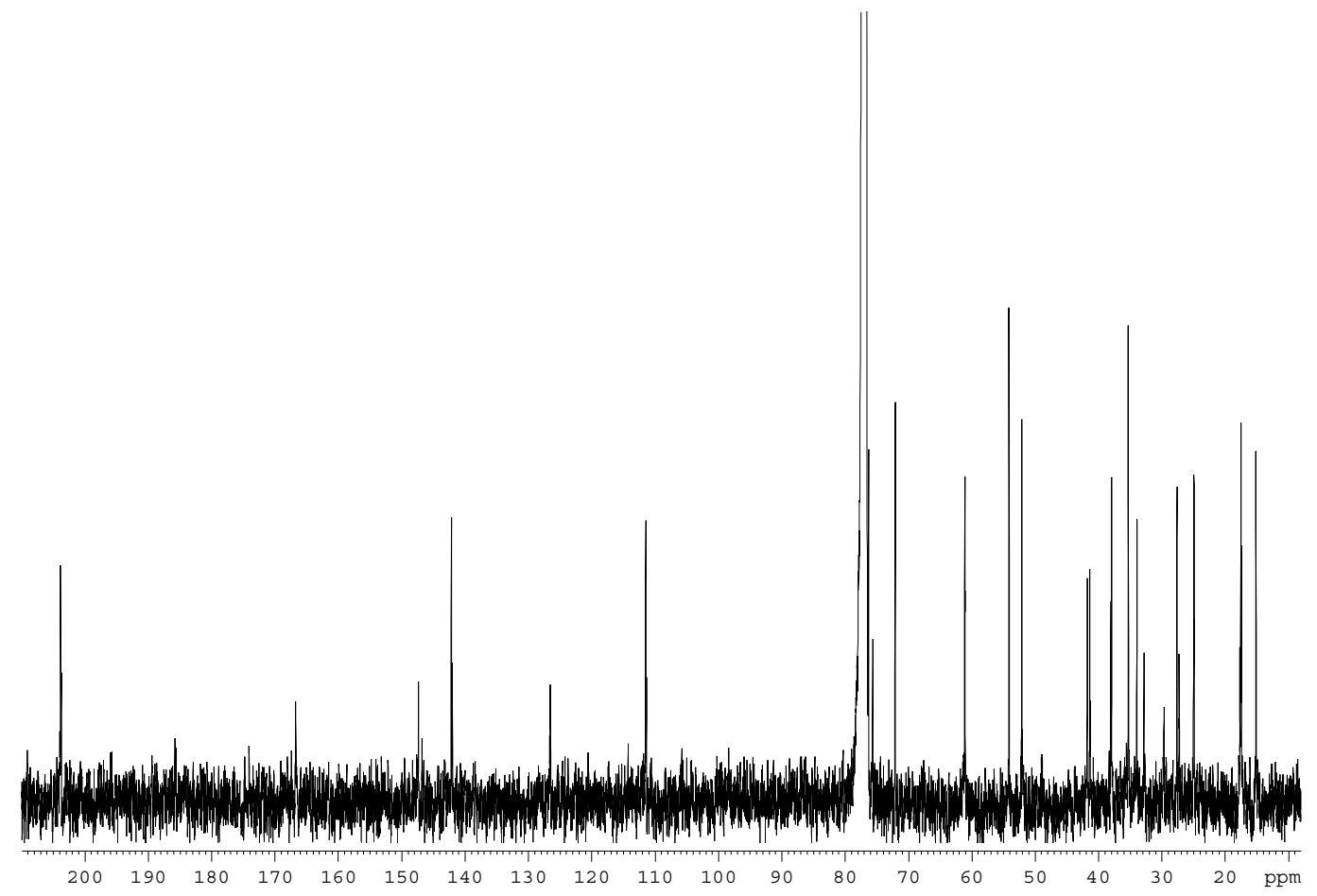


Figure S16. ${ }^{1} \mathrm{H}-\mathrm{NMR}$ spectrum of negombin $\mathrm{H}\left(\mathbf{8} ; 400 \mathrm{MHz}, \mathrm{CDCl}_{3}\right)$

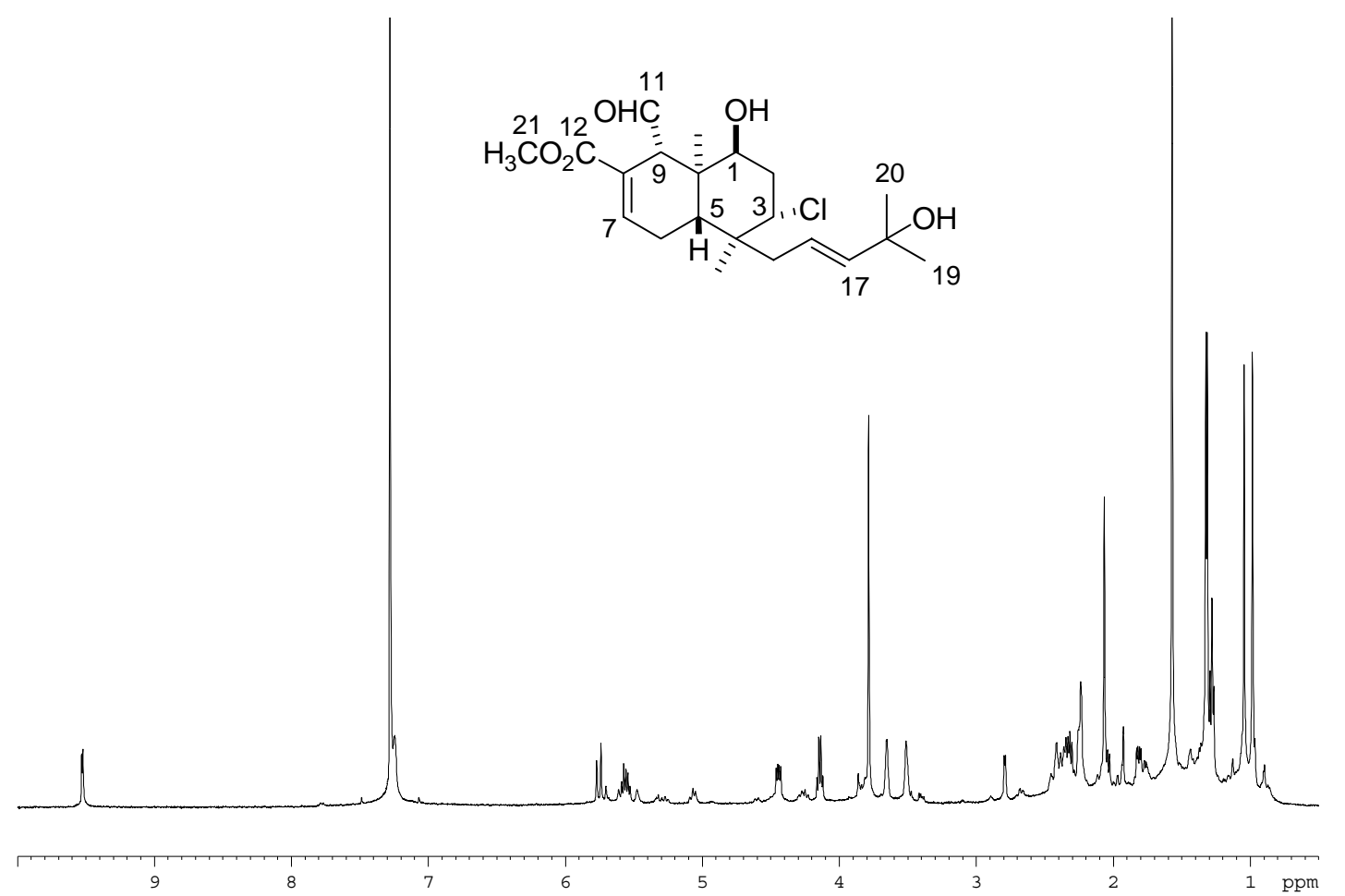

Figure S17. ${ }^{13} \mathrm{C}-\mathrm{NMR}$ spectrum of negombin $\mathrm{H}\left(\mathbf{8} ; 100 \mathrm{MHz}, \mathrm{CDCl}_{3}\right)$

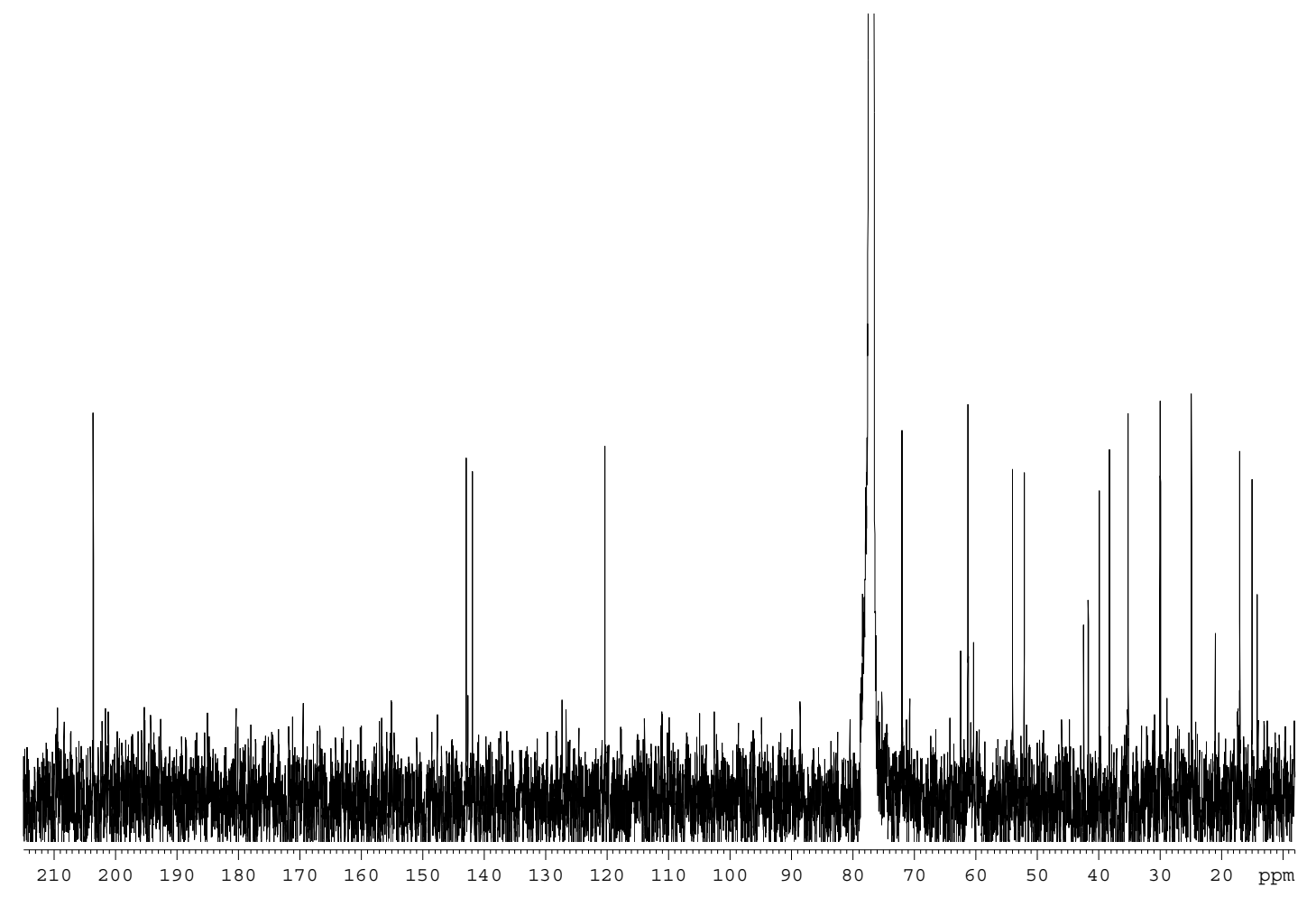


Figure S18. ${ }^{1} \mathrm{H}-\mathrm{NMR}$ spectrum of negombin I (9; $\left.500 \mathrm{MHz}, \mathrm{CDCl}_{3}\right)$

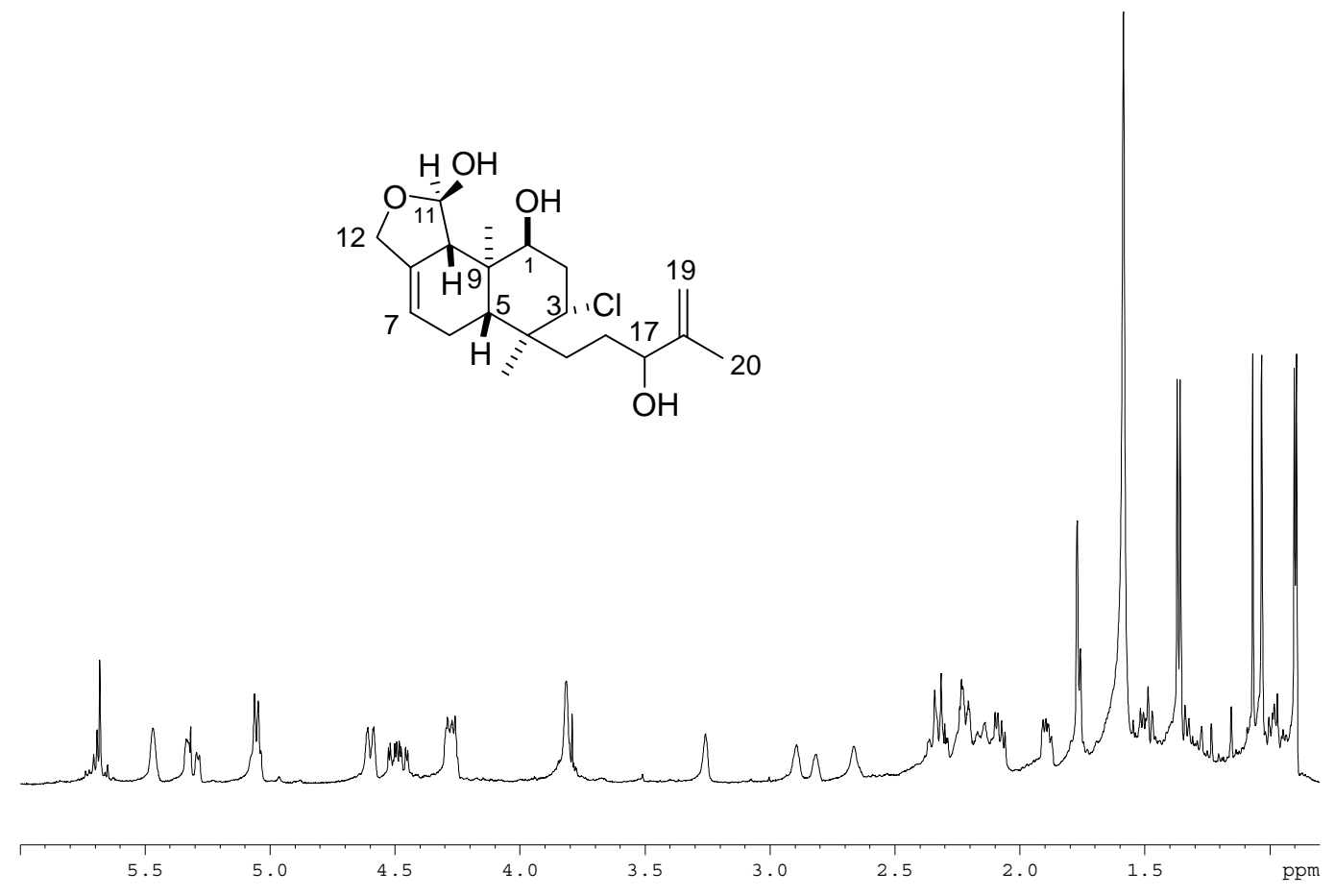

Figure S19. HSQC spectrum of negombin I $\left(\mathbf{9} ; 500 \mathrm{MHz}, \mathrm{CDCl}_{3}\right)$

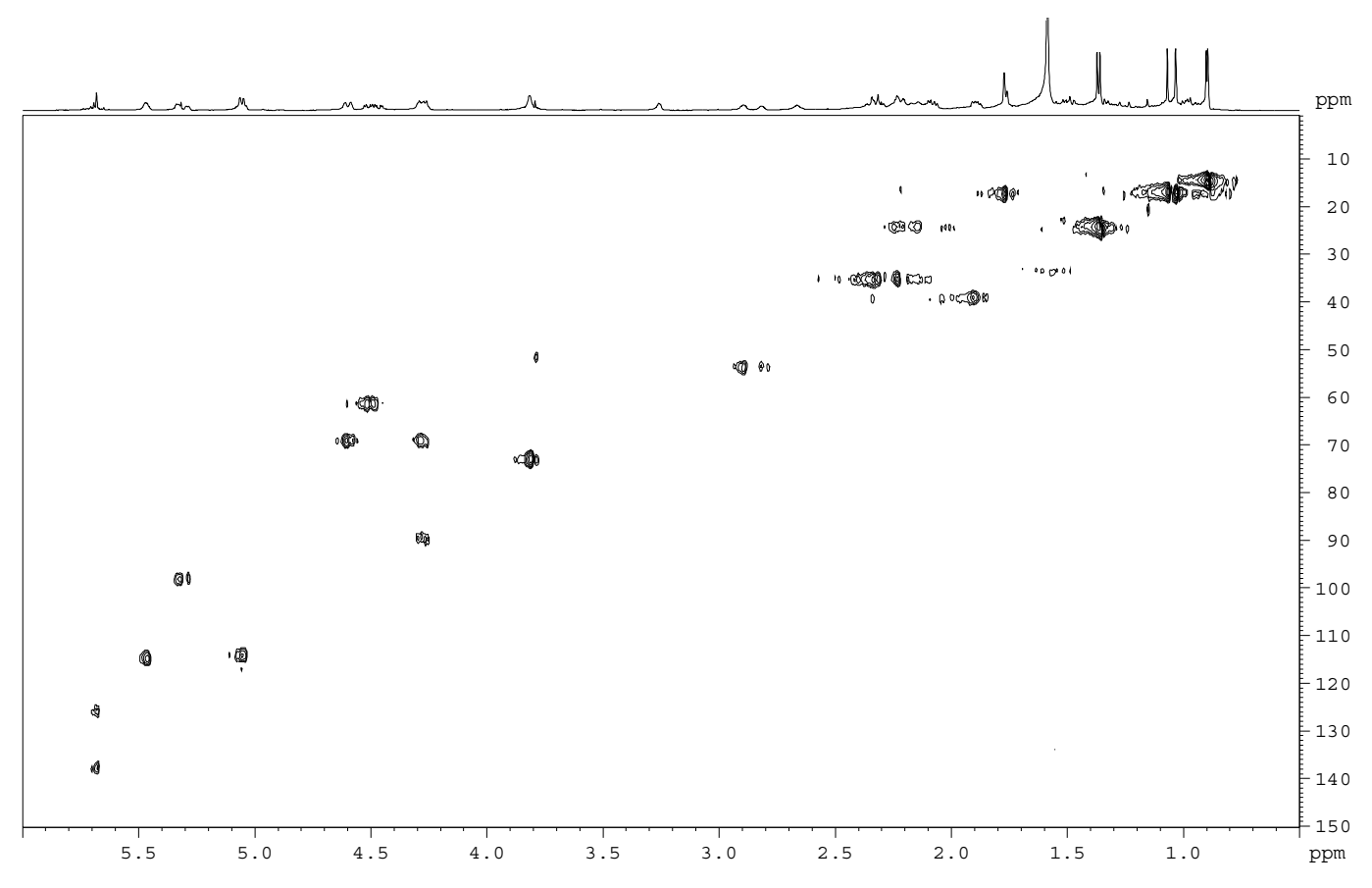

